\title{
Navigation in REVERIE's Virtual Environments
}

\author{
Fiona M. Rivera ${ }^{\dagger}$ \\ Queen Mary \\ University of London
}

\author{
Fons Fuijk* \\ CWI Amsterdam
}

\author{
Ebroul Izquierdo ${ }^{\ddagger}$ \\ Queen Mary \\ University of London
}

\begin{abstract}
This work presents a novel navigation system for social collaborative virtual environments populated with multiple characters. The navigation system ensures collision free movement of avatars and agents. It supports direct user manipulation, automated path planning, positioning to get seated, and follow-me behaviour for groups. In follow-me mode, the socially aware system manages the mise en place of individuals within a group. A use case centred around on an educational virtual trip to the European Parliament created for the REVERIE FP7 project, also serves as an example to bring forward aspects of such navigational requirements.
\end{abstract}

Keywords: Virtual reality, 3D navigation, Avatars, 3D world.

Index Terms: H.5.1 [Information Interfaces and Presentation]: Multimedia Information Systems-Artificial, augmented, and virtual realities;

\section{INTRODUCTION}

Designing navigation systems intended for multiple users within complex virtual spaces is not a trivial task. The presented system has been developed for such a case, as part of the EC $7^{\text {th }}$ Framework REVERIE project: REal and Virtual Engagement in Realistic Immersive Environments [1]. The navigation system has been implemented in a REVERIE use case scenario aimed at, but not limited to, the educational sector [2]. Real-world teachers and students can engage as a social group in a virtual educational field trip to a place of cultural significance. The teacher and students are embodied as avatars in a virtual version of the European Parliament Assembly Chamber in Brussels (See Figure 1). Similar to a real-world tour with a personal tour guide, the virtual tour is hosted by a knowledgeable tour guide: a software-driven intelligent virtual autonomous agent. The goal in the presented navigation system is to allow for a mixture of types of movement. This includes via a group of avatars following a leading agent, plus some degree of individual freedom of movement.

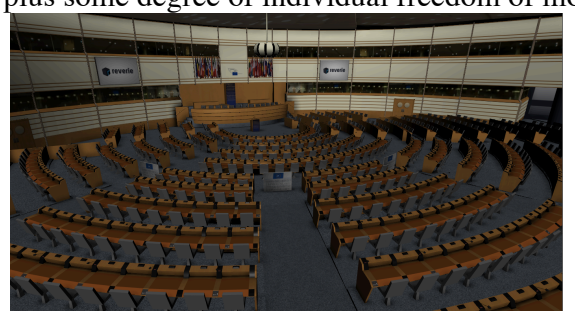

Figure 1: Virtual European Parliament Assembly Chamber

\footnotetext{
†f.m.rivera@qmul.ac.uk

*fons.kuijk@cwi.nl

‡e.izquierdo@qmul.ac.uk
}

\section{NaVigation System}

Common algorithms for autonomous path planning are methods such as potential fields [3], graph search methods [4, 5], and Evolutionary Algorithmic (EA) techniques [6,7]. Of these the EA-based methods seem well suited for dynamic environments as they can deal with uncertainty in location, however they tend to be somewhat inefficient. The Explicit Corridor Map (ECM) method [8] is more efficient for our purpose as it can create believable paths of different characteristics, and algorithms are fast and simple as our results show. This method is also well suited for steering many characters simultaneously. The navigation system presented here, is therefore based on the ECM library ${ }^{1}$, however it has been enhanced in this work to include accommodation for seating, end placement facing (orientation) and automatic positioning of a group (optionally based on levels of engagement).

The REVERIE system offers user controlled navigation through Map navigation: the user clicks on a target position within a top view image of the virtual room (see Figure 2 left). The Explicit Corridor Map (ECM) provides the basic indicative route planning and dynamic collision detection to avoid possible collisions with other characters or moving obstacles crossing the indicative route.

Due to the large size of the virtual room, combined with the lack of free space in-between rows of desks, users ${ }^{2}$ found it challenging to click precisely enough on an accessible position in the top view image. Attempts to manoeuvre close to desks were rejected when they were seen as attempts to reach an inaccessible area. To avoid such rejects, the system was enhanced to replace an inaccessible target position by a nearest accessible position.

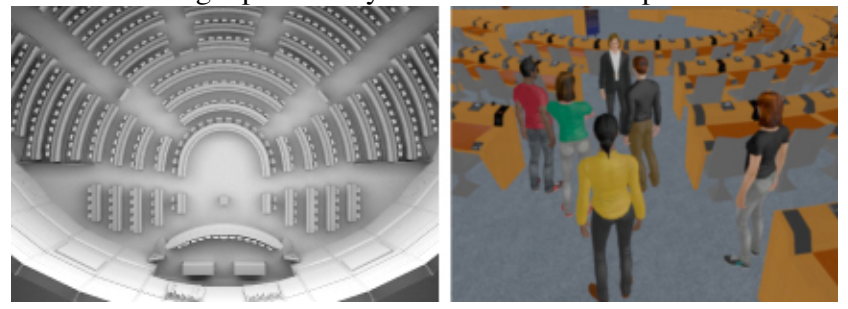

Figure 2: Top view map navigation image (left). Avatars in followme mode facing the agent in the parliament (right).

The system was also enhanced to allow for users to indicate the desired end placement orientation (facing) once they reach a destination. It takes the mouse-pressed target location on the map as destination, and the mouse-released location as an indicator of direction. In this way a one click-drag-release specifies both destination and orientation.

One requirement was that students, embodied as avatars, have to be seated to participate in debates. Therefore the REVERIE navigation system accepts locations on or close to a seat location and interprets that as a signal: "I want to be seated". We enhanced the system with data regarding seat positions. Three $2 \mathrm{D}$ vectors describe each seat: location, access point and direction. The ECM algorithm cannot produce an indicative route based on the seat

\footnotetext{
${ }^{1}$ http://www.staff.science.uu.nl/ gerae101/motion_planning/ecm.html

2 users testing the system informally in the development stages
} 
location itself, since that is an inaccessible point. The indicative route is calculated by replacing the inaccessible seat location with the seat access point: an accessible point near the seat. The navigation will bring the avatar up to this access point and turn the avatar in the seat direction, ready to be seated. When the avatar requires navigating away from the seat, the same access point is taken as start of the next indicative route.

The system supports fine-grained navigation: users can use the keyboard to reorient the character on the spot, or to make step-bystep adjustments to their positions. When adjusting position, the system first checks if the small steps to take in any of four directions on the keyboard will cause a collision; first with static obstacles, and next with dynamic objects. If the route is clear, the character is transported to that new position in a smooth way, taking a specified number of frame-times.

In Follow-Me mode, destinations of individuals of a group are assigned by the system based on the destination and orientation of a leading agent. The system automatically assigns destinations within a space in front of and facing agent. Minimal distance from the agent, and the average clearance between individual members of the group and other obstacles is specified by parameters. Filling the space starts at the minimal distance from the agent, and as more avatars are added, this distance increases. The density distribution in the space in front of the agent decreases as distance increases. Examples of this automated mise-en-place are shown in Figure 2 (right). Being positioned in Follow-Me mode users can still manipulate their avatar, either by keyboard or by map navigation to obtain a preferred view, or to be near to a friend.

The REVERIE platform also has components that infer engagement of the user [2]. The automatic assignment of positions can be prioritized based on the level of engagement. As a result, similar to the real-world, more engaged participants may obtain positions nearer to the agent (the tour guide) than the less engaged participants. This level of engagement can also influence the assigned walking speed.

\section{Preliminary Results}

The navigation system has been initially tested with a small group of 5 avatars and an agent on a 64-bit Windows 7 configuration with an Intel® ${ }^{\circledR}$ Core $^{\mathrm{TM}}$ i7-2600 CPU @ $3.40 \mathrm{GHz}$, 16 GB RAM and a Radeon ${ }^{\mathrm{TM}}$ R9 280X graphics card. In testing, the group followed the agent from a starting position near the entrance, then manoeuvred through a narrow pathway, to end up at the central area in front of the lectern. Comparisons were made between the navigation times against the full frame time needed for rendering (Figure 3).

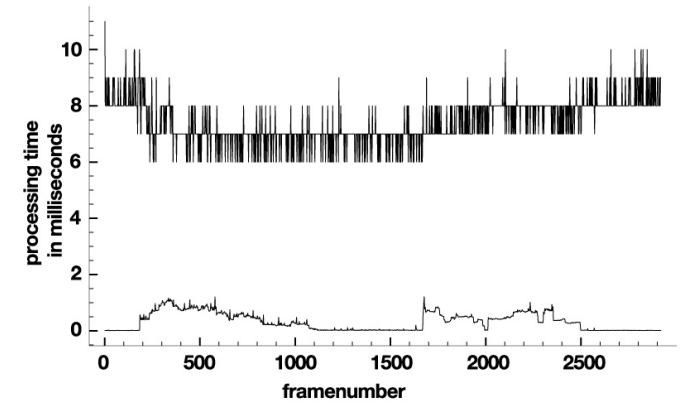

Figure 1: Navigation time (lower curve) compared to frame time for navigation of the group (upper curve).

Our preliminary results show that navigation time does not exceed $1.2 \mathrm{~ms}$ at all times. The relatively high computational time at the beginning reflects navigation before entering the narrow pathway; each character has to deal with position and speed of all others. Around the middle of the graph the group walks down the pathway where the computational complexity has reduced (each of the characters only has to avoid colliding with its forerunner). When the group reaches the spacious middle sector, each character has to find its way to its final destination, possibly having to deal with position and speed of all other characters again, as reflects in an increase of computation time.

For a single character navigating with no dynamic obstacles to deal with we found navigation times of less than $0.04 \mathrm{~ms}$ on average whereas navigating through a group (that forces the character to leave the indicative route) led to navigation times below $0.6 \mathrm{~ms}$.

\section{Conclusions and Future Work}

Our navigation system has been developed in the context of the REVERIE platform to ensure collision free movement of avatars and agents. It accounts for path planning for individuals, followme behavior for groups, and manages the mise en place of individuals within a group, optionally taking into account user engagement. The system has been shown to be efficient in preliminary small group tests. Further field trials with larger groups are planned. In a future version we also hope to support social aware assignment to prioritize placement near a friend.

\section{ACKNOWLEDGEMENTS}

The research leading to these results has received funding from the European Community's Seventh Framework Programme (FP7/2007-2013) under grant agreement no. ICT-2011-7-287723 (REVERIE project).The navigation system is build on top of the ECM library made available by the University of Utrecht, Department of Information and Computer Sciences.

\section{References}

[1] http://www.reveriefp7.eu

[2] Wall, J., Izquierdo, E., Argyriou, L., Monaghan, D.S., O’Connor, N.E., Poulakos, S., Smolic, A., Mekuria, R., 2014, "REVERIE: Natural Human Interaction in Virtual Immersive Environments", Proc. of IEEE International Conference on Image Processing (ICIP) 2014

[3] Khatib, O., 1986, "Real-Time Obstacle Avoidance for Manipulator and Mobile Robots", International Journal of Robotics Research, Vol. 5, No. 1, pp. 90-98.

[4] Mitchell, J.S.B., and D.M. Keirsey, 1984, "Planning Strategic Paths through Variable Terrain Data", Proc. of the SPIE Conference on Applications of Artificial Intelligence, Vol. 485, Arlington, VA, 1984, pp. 172-179.

[5] Stentz, A., 1994, "Optimal and Efficient Path Planning for PartiallyKnown Environments", Proc. of the 1994 International Conference on Robotics and Automation, Vol. 4, Los Alamitos, CA., pp. 33103317.

[6] Fogel, D.B., and L.J. Fogel, 1990, “Optimal Routing of Multiple Autonomous Underwater Vehicles through Evolutionary Programming, Proc. of the 1990 Symposium on Autonomous Underwater Vehicle Technology, Washington, D.C., pp. 44-47.

[7] Capozzi, B.J., and J. Vagners, 2001, "Evolving (Semi)-Autonomous Vehicles", Proc. of the 2001 AIAA Guidance, Navigation and Control Conference, Montreal, Canada.

[8] Geraerts, R., Planning Short Paths with Clearance using Explicit Corridors. In IEEE International Conference on Robotics and Automation, pp. 1997-2004, 2010 